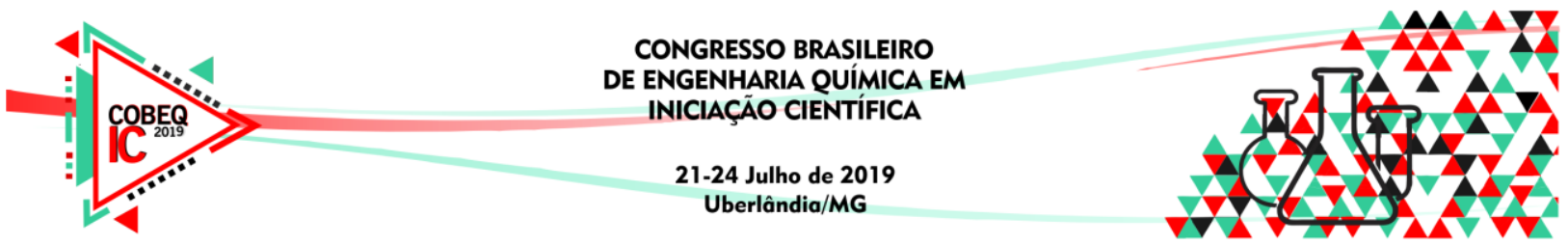

\title{
CARACTERIZAÇÃO FÍSICO-QUÍMICA DE RESÍDUOS INDUSTRIAIS PARA APROVEITAMENTO ENERGÉTICO
}

\author{
S. S. PLÁCIDO JÚNIOR ${ }^{1}$, J. G. DE ALBUQUERQUE² ${ }^{2}$ S.L.F. ANDERSEN ${ }^{1}$ \\ ${ }^{1}$ Universidade Federal da Paraíba, Departamento de Engenharia de Energias Renováveis \\ ${ }^{2}$ Universidade Federal da Paraíba, Departamento de Engenharia Química \\ E-mail para contato: sidney.junior@ cear.ufpb.br
}

\begin{abstract}
RESUMO - A energia proveniente da biomassa está cada vez mais presente no cenário energético nacional ao longo dos últimos anos. Em parte, esse crescimento ocorre em resposta a um dos principais entraves enfrentados pela indústria, que diz respeito aos seus resíduos sólidos. Cada vez mais procuram-se formas de reutilização dos respectivos resíduos, uma vez que aterros industriais demandam de uma grande área e geram impactos ao ambiente no qual são instalados. $\mathrm{O}$ objetivo deste trabalho é caracterizar físico-quimicamente por meio de análise elementar, análise imediata, poder calorífico, composição química das cinzas, e análise termogravimétrica (TGA/DTG), resíduos sólidos industriais, sendo eles: o lodo da estação de tratamento de efluentes de uma indústria têxtil (LT), a semente de acerola (SA) e a borra de algodão (BA). As análises apresentaram teor de cinzas entre $2,48 \%$ e $51,81 \%$; teor de material volátil entre $44,72 \%$ e $85,40 \%$; carbono fixo variando de 3,32\% a 22,43\%; e PCS na faixa de 9,56 $\mathrm{MJ}^{\mathrm{kg}} \mathrm{kg}^{-1}$ a 21,90 MJ.kg-1. Interpretando os resultados obtidos e comparando-os com a literatura, foi possível concluir que, dentre as amostras citadas, SA e BA são mais promissoras para o uso em processos de conversão térmica.
\end{abstract}

\section{INTRODUÇÃO}

Devido ao desenvolvimento industrial e comercial das últimas décadas ser acompanhado por um rápido processo de urbanização e aumento populacional, tanto o comércio quanto a população em geral têm elevado sua procura por matérias-primas, produtos e energia, acarretando num aumento na geração de resíduos. Tais resíduos quando não recebem tratamento adequado, comprometem a qualidade do ambiente onde são depositados (DIAS JÚNIOR, 2013). Segundo DIAS JÚNIOR (2013) uma das indústrias que mais contaminam o ambiente, por gerar grande quantidade de resíduos, é a têxtil, uma vez que uma indústria de lavanderia têxtil de médio porte gera em média 6 toneladas de lodo seco ou 120 toneladas de lodo com $95 \%$ de umidade, por ano.

Outro resíduo com que se deve ter atenção é o proveniente da acerola, já que, no Brasil, a produtividade média de 29,65 toneladas por hectare ao ano acarretam em $40 \%$ do volume de produção sendo constituído por sementes e bagaço (MARQUES, 2013). 


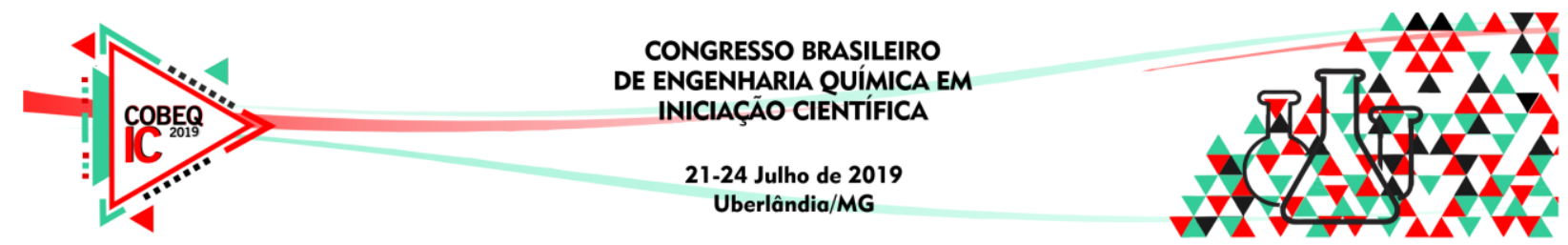

A Lei $n^{\circ} 12.305$ de 2 de agosto de 2010, institui a Política Nacional de Resíduos Sólidos (BRASIL, 2010), a qual reúne um conjunto de princípios, objetivos, instrumentos, diretrizes, metas e ações adotados pelo Governo Federal objetivando a gestão integrada e o gerenciamento ambientalmente adequado dos resíduos sólidos, fazendo com que os produtores e empresas envolvidas busquem alternativas de utilização deste tipo de material, como por exemplo, a transformação em energia (JACINTO, 2017), sendo esta transformação o foco do presente estudo.

O objetivo do estudo consiste na caracterização físico-química de determinados resíduos sólidos industriais com a finalidade de determinar uma potencial forma de utilização destes resíduos para aproveitamento energético, por meio de processos termoquímicos, tais como combustão, pirólise e gaseificação.

\section{MATERIAL E MÉTODOS}

As amostras selecionadas para este trabalho foram: lodo do tratamento de efluentes de uma indústria têxtil (LT), semente de acerola (SA) e borra de algodão (resíduo da extração do óleo) (BA). Todas as amostras utilizadas durante a pesquisa, foram cedidas por indústrias instaladas nos estados da Paraíba e Pernambuco.

Inicialmente as amostras LT e SA foram secas em uma estufa de circulação de ar e posteriormente moídas e peneiradas com uma peneira granulométrica de 100 mesh. A amostra BA foi utilizada na forma de base úmida por estar em estado pastoso.

A análise elementar foi realizada no equipamento Perkin Elmer Modelo 2400 Series II CHNS/O com uso de reagente EA6000, onde a amostra passou por um processo de combustão completa e os gases resultantes desse processo foram analisados por um cromatógrafo. O teor de oxigênio $(\mathrm{O})$ foi determinado por diferença conforme Equação 1:

$O=100-(C+H+N+S)$

A análise imediata foi realizada para determinar o teor de umidade (\%U), teor de cinzas $(\% \mathrm{C})$, materiais voláteis $(\% \mathrm{~V})$ e carbono fixo $(\% \mathrm{CF})$ das amostras, segundo as normas ASTM E1755 e ASTM E872. Todas as análises foram feitas pelo menos em duplicata. Para determinação do \%U, foi utilizado uma balança de umidade AND MF-50. Para a obtenção do teor de carbono fixo, foi utilizada a Equação 2:

$\% C F=100-(\% C+\% V)$

Para determinar o poder calorífico superior (PCS), foi utilizada uma bomba calorimétrica IKA C200, seguindo a norma ASTM D5865. O valor do poder calorífico inferior (PCI) foi obtido por meio da Equação 3; Onde \%H corresponde ao teor de hidrogênio determinado pela análise elementar.

$$
P C I=P C S-\left[600 \cdot\left(\frac{9 . \% H}{100}\right)\right]
$$


A análise termogravimétrica (TGA/DTG) foi realizada utilizando o equipamento da marca TA, modelo Q-50. A taxa de aquecimento foi de $10{ }^{\circ} \mathrm{C} \cdot \mathrm{min}^{-1}$ da temperatura ambiente até $900{ }^{\circ} \mathrm{C}$, em atmosfera inerte $\left(\mathrm{N}_{2}\right.$, pureza mínima 99,996\%) a um fluxo de $100 \mathrm{~mL} \cdot \mathrm{min}^{-1}$, com massa de aproximadamente $10 \mathrm{mg}$.

Para a caracterização química das cinzas das amostras foi utilizado a espectroscopia de fluorescência de raios X (FRX), em espectrômetro modelo FRX 1800, de marca Shimadzu. Este tipo de caracterização é importante para determinar a composição mineral das amostras, uma vez que nelas pode conter substâncias que em concentrações relevantes, apresentem ponto de fusão baixo $\left(<800^{\circ} \mathrm{C}\right)$ (LIRA et al.,2014).

\section{RESULTADOS E DISCUSSÕES}

A Tabela 1 apresenta os resultados das caracterizações físico-químicas dos resíduos.

Tabela 1 - Caracterização Físico-Química dos Resíduos Sólidos

\begin{tabular}{|c|c|c|c|}
\hline Amostras & $\mathrm{LT}^{*}$ & $\mathrm{SA}^{*}$ & $\mathrm{BA}^{\mathrm{b}}$ \\
\hline Umidade $^{\mathrm{a}}$ & $7,22 \pm 1,14$ & $9,83 \pm 0,22$ & - \\
\hline Cinzas $^{a}$ & $51,81 \pm 0,97$ & $2,48 \pm 0,16$ & $11,29 \pm 0,10$ \\
\hline Voláteis $^{\mathrm{a}}$ & $44,72 \pm 2,49$ & $75,09 \pm 0,40$ & $85,40 \pm 1,20$ \\
\hline Carbono Fixo $^{\mathrm{a}}$ & $3,47 \pm 0,00$ & $22,43 \pm 0,00$ & $3,32 \pm 0,00$ \\
\hline $\mathrm{PCS}^{\mathrm{c}}$ & $9,56 \pm 0,02$ & $19,02 \pm 0,07$ & $21,90 \pm 0,24$ \\
\hline $\mathrm{PCI}^{\mathrm{c}, \mathrm{e}}$ & 7,76 & 15,78 & 17,58 \\
\hline$C^{d}$ & 20,76 & 47,66 & 45,69 \\
\hline $\mathrm{H}^{\mathrm{d}}$ & 3,34 & 6,00 & 8,00 \\
\hline $\mathrm{N}^{\mathrm{d}}$ & 6,25 & 2,27 & 0,70 \\
\hline $\mathrm{S}^{\mathrm{d}}$ & 0,69 & 1,15 & 1,43 \\
\hline $\mathrm{O}^{\mathrm{d}, \mathrm{f}}$ & 68,96 & 42,92 & 44,18 \\
\hline \multicolumn{4}{|c|}{ Composição química das cinzas ${ }^{a}$} \\
\hline $\mathrm{SiO}_{2}$ & 84,00 & 1,64 & 0,41 \\
\hline $\mathrm{Al}_{2} \mathrm{O}_{3}$ & 5,24 & - & 0,25 \\
\hline $\mathrm{MgO}$ & 4,02 & 6,11 & 4,86 \\
\hline $\mathrm{P}_{2} \mathrm{O}_{5}$ & 1,89 & 28,23 & 10,14 \\
\hline $\mathrm{SO}_{3}$ & 1,50 & 8,57 & 0,43 \\
\hline $\mathrm{Na}_{2} \mathrm{O}$ & 1,23 & - & 79,68 \\
\hline $\mathrm{CaO}$ & 1,10 & 22,98 & 0,40 \\
\hline $\mathrm{Fe}_{2} \mathrm{O}_{3}$ & 0,45 & 3,04 & 0,31 \\
\hline $\mathrm{K}_{2} \mathrm{O}$ & - & 25,30 & 2,77 \\
\hline $\mathrm{Cl}$ & - & 0,70 & 0,55 \\
\hline Outros & 0,58 & 3,42 & 0,19 \\
\hline
\end{tabular}




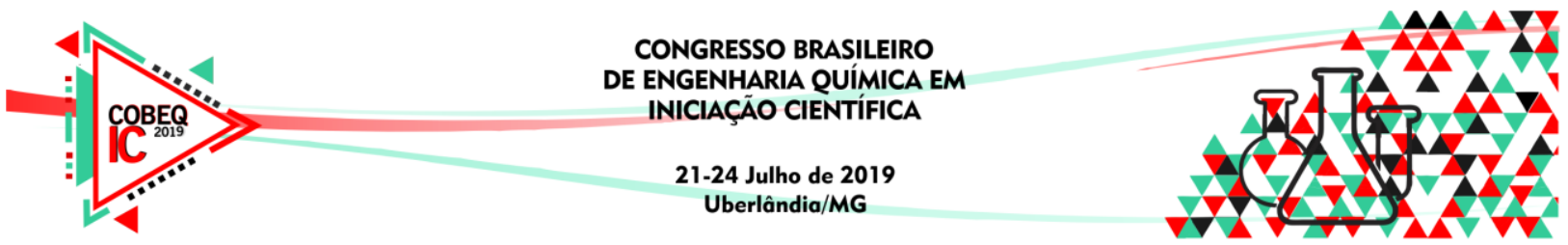

Nela é possível identificar que a amostra LT possui um alto teor de cinzas, $51,81 \%$, podendo ser prejudicial em processos de conversão termoquímica, uma vez que representa uma menor quantidade de combustível na caldeira, resultando em um menor poder calorífico (JACOME, 2014), condizendo com os resultados encontrados durante a análise. Unindo estes dados ao fato de a composição química de $\mathrm{LT}$ conter $84 \%$ de $\mathrm{SiO}_{2}, 5,24 \%$ de $\mathrm{Al}_{2} \mathrm{O}_{3}$, que são elementos inorgânicos, fazem com que o uso de LT para fins energéticos em processos termoquímicos não possua muitas vantagens. Além disso, LT apresenta um baixo poder calorífico superior se comparado com SA, BA e com amostras da literatura, como por exemplo, a Eucalyptus grandis e a casca de coco que possuem PCS, respectivamente, de 19,02 MJ.kg-1 21,90 MJ.kg-1, 17,895 MJ.kg-1 (MÜZEL, et al., 2014) e 20,51 MJ.kg-1 (TSAMBA et al., 2006).

Por outro lado, SA apresenta baixa percentagem de cinzas, $2,48 \%$ e um alto teor de voláteis, $75,09 \%$ que junto com seu alto teor de carbono fixo contribui a uma relação MV/CF próxima àquela para carvões (CONSUEGRA, 2013), o que confirma seu potencial de uso como fonte de energia. Outro fato que auxilia nessa utilização diz respeito à composição química de suas cinzas, uma vez que possui $25,30 \%$ de $\mathrm{K}$ e $28,23 \%$ de $\mathrm{P}_{2} \mathrm{O}_{5}$. Porém deve-se observar que esses compostos baixam a temperatura de fusão das cinzas podendo acarretar em problemas operacionais dependendo da temperatura do sistema.

BA apresentou resultados satisfatórios em termos de PCS, 21,90 MJ. $\mathrm{kg}^{-1}$ e teor de voláteis, $85,40 \%$. Além disso, os valores obtidos com a análise elementar, como pode ser visto na Tabela 1, indicaram que a amostra possui valores aproximados aos das biomassas normalmente utilizadas para aproveitamento energético, como o bagaço da cana-de-açúcar, o qual apresenta valores entre 45,5-50,3\% para o carbono, 4,6-6,3\% para o hidrogênio, 0,130,40\% para o nitrogênio e 43,1-49,5\% para o oxigênio (DA SILVA, 2017), fazendo da borra uma opção a ser considerada.

A Figura 1 mostra as curvas de TGA e DTG para a semente de acerola, borra de algodão e lodo, respectivamente. A partir das curvas é possível observar que SA apresentou três principais perdas mássicas: 5,69\% referente à perda de umidade residual, $19,95 \%$ referente à decomposição do material volátil de menor peso molecular e 40,86\% referente à decomposição do material volátil de maior peso molecular onde há o maior pico na DTG com uma velocidade de perda de massa de $0,54 \mathrm{mg} \cdot \mathrm{min}^{-1}$ na temperatura de $347^{\circ} \mathrm{C}$. Em relação à BA é verificado duas perdas principais: $18,02 \%$ referente à perda por umidade juntamente com material volátil de maior peso molecular, uma vez que a curva DTG apresenta seu maior pico com uma velocidade de perda de massa de $0,98 \mathrm{mg} \cdot \mathrm{min}^{-1} \mathrm{em}$ temperatura de $126{ }^{\circ} \mathrm{C}$. A segunda perda é caracterizada pelo material volátil de menor peso molecular, sendo $37,99 \%$. Tais dados mostram que a borra é uma biomassa muito reativa. Próximo a $900{ }^{\circ} \mathrm{C}$, a curva DTG da acerola e borra voltam a subir, indicando a possível volatilização da lignina residual (BURHENNE et al., 2013). 


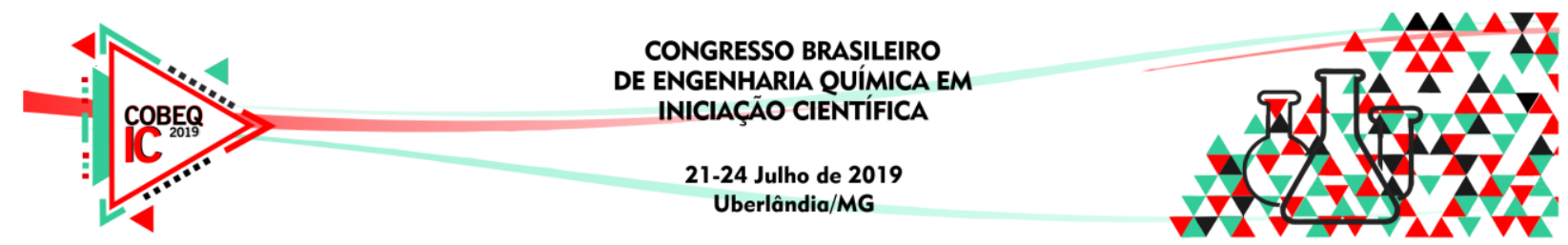

Figura 1 - TGA/DTG dos Resíduos Sólidos.
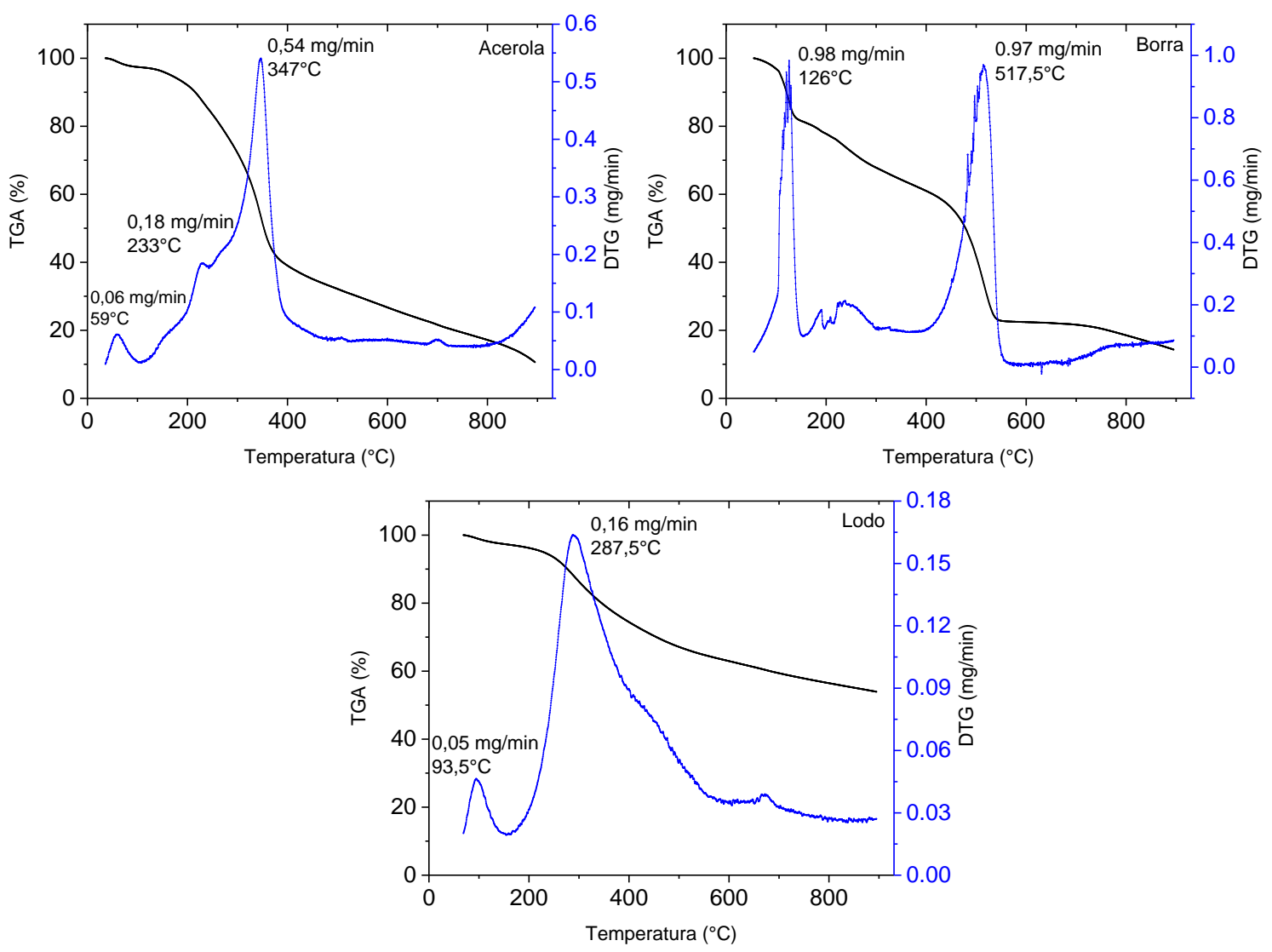

LT apresentou duas perdas significativas de massa: 3,31\% referente à perda de umidade residual e $32,46 \%$ referente decomposição do material volátil onde há uma alta taxa de perda de massa $\left(0,16 \mathrm{mg} \cdot \mathrm{min}^{-1}\right)$ na temperatura de pico de $287,5^{\circ} \mathrm{C}$. Comparando os resultados obtidos com os valores encontrados na análise imediata, pode-se comprovar o alto teor de cinzas presente na amostra em questão.

\section{CONCLUSÃO}

A caracterização físico-química das biomassas permitiu constatar que a SA e a BA mostraram, teor de cinzas e PCS comparável ao de biomassas comumente utilizadas em processos de conversão energética como o Eucalyptus grandis e a casca do coco apresentando potencial para serem utilizados como fonte de energia em processos de conversão termoquímicos. Contudo, o LT não apresenta potencial para uso individual em processos termoquímicos devido ao seu alto teor de cinzas, baixo teor de materiais voláteis e baixo PCS. 


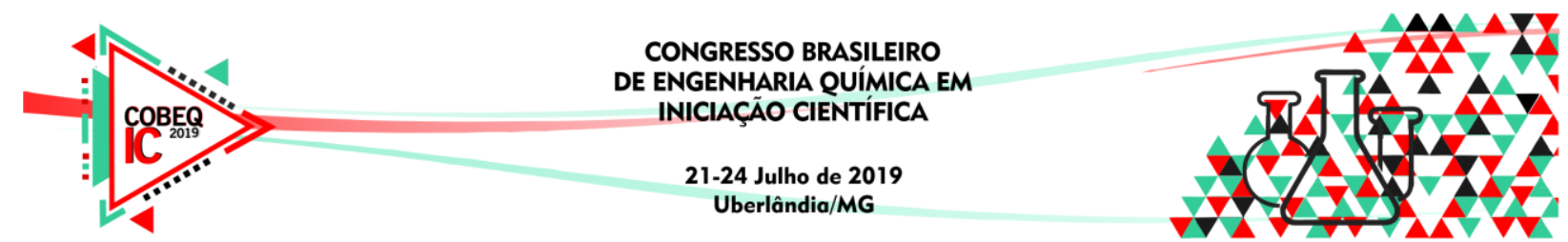

\section{REFERÊNCIAS BIBLIOGRAFICAS}

ASTM. E1755. Standard Test Method For Ash In Biomass., 1984.

ASTM. E872. Standard Test Method For Volatile Matter In The Analysis Of Particulate Wood Fuels., 2013.

BRASIL. Lei $\mathrm{n}^{\mathrm{o}}$ 12.305, de 2 de agosto de 2010. Institui a Política Nacional de Resíduos Sólidos. Diário Oficial da República Federativa do Brasil, Brasília, DF, 2 ago. 2010. Disponível em: <(http://www.planalto.gov.br/ccivil_03/_ato20072010/2010/lei/112305.htm)> Acesso em 02 de abril de 2019.

BURHENNE, L.; MESSMER, J.; AICHER, T.; LABORIE, M. P. The Effect Of The Biomass Components Lignin, Cellulose And Hemicellulose On TGA And Fixed Bed Pyrolysis. Journal Of Analytical And Applied Pyrolysis, v. 101, p. 177-184, 2013.

CONSUEGRA, A. D. S. A.; Cinética Da Gaseificação Do Bagaço De Laranja E Serragem De Madeira Com Co2, 2013.

DA SILVA, J. C. G.; Estudo Dos Parâmetros Cinéticos Da Pirólise Do Bagaço De Cana-deaçúcar, 2017.

DIAS JUNIOR, M. L.; Incorporação De Lodo Têxtil Em Blocos Cerâmicos, 2013.

JACINTO, R. C.; Produção De Pellets Para Energia Usando Diferentes Resíduos De Biomassa Agrícolas E Florestais, 2017.

JACOME, D. L. F.; Caracterização Físico-química das Cinzas de Palha de Cana-de-Açúcar Através de Análises Térmicas Simultâneas (STA), 2014.

LIRA, J. O. B.; SILVA, K. C. G; ANDERSEN S. L. F. Estudo Das Características De Combustão De Resíduos Sólidos Da Região Nordeste Utilizando Análise Termogravimétrica. XX Congresso de Engenharia química. Área temática: Engenharia Ambiental e Tecnologias Limpas. Florianópolis-SC. 2014.

MARQUES, T. R.; Aproveitamento Tecnológico de Resíduos de Acerola: Farinhas e Barras de Cereais, 2013.

MÜZEL, S. D. Et Al, Poder Calorífico Da Madeira De Eucalyptus Grandis E Da Hevea Brasiliensis. Brazilian Journal Of Biosystems Engineering, v. 8, n.2, p. 166-172, 2014.

TSAMBA, A. J.; YANG, W.; BLASIAK, W. Pyrolysis Characteristics And Global Kinetics Of Coconut And Cashew Nut Shells. Fuel Processing Technology. v. 87. p. 523-530. jun. 2006. 